\title{
11. On the Tokyo-Mitaka Catalogue of Zenith Stars and Its Catalogue Difference.
}

\author{
By Kônosuke Tuzi. \\ Tokyo Astronomical Observatory. \\ (Comm. by Y. Hagihara, M.J.A., March 13, 1950.)
}

The observation of the right ascensions of 500 zenith stars has been carried out by the writer with a Bamberg transit instrument in the Tokyo Astronomical Observatory. The proper motions of those stars were determined and a star catalogue has been compiled on the basis of this observation. The method of procedure and the brief summary of the result are reported in the present note.

\section{The zenith stars and the system of reference.}

The knowledge of the precise positions of the stars in the zenith zone of our observatory is fundamentally important as a photographic zenith tube is now being constructed and will be in use in near future. With this instrument, we may observe a group of faint stars in the limitted zone of the zenith, while the positions of such faint stars have not been determined with sufficient precision in the past.

The present catalogue contains the observed positions of zenith stars which are to be adopted as the reference points for determining the positions of those faint zenith stars.

In our observation, 100 stars in the zenith zone contained in the FK3 catalogue have been adopted as the clock stars, and all of the observed right ascensions have been reduced to the FK3 system adopting Newcomb's astronomical constants.

2. The observations of the right ascensions of the zenith stars.

The detail of the instrument, the method of observation and the individual result for each night have been described in the Tokyo-Mitaka Catalogue of Zodiacal Stars published in the Annals of our observatory ${ }^{1)}$. The accuracy of the observation has been examined every night by computing the probable error of the clock correction of the time-keeper directly used for the observation. The mean value of the probable errors of the clock corrections for a transit of a single star is \pm 0.02 .

The observation was commenced in September 1944 and completed in August 1948. The program stars were observed 10 to 40 times, and the probable error of the observed right ascension amounts $\pm 0.002 \sim \pm 0.007$.

1) Annals of the Tokyo Astronomical Observatory, Second Series, Vol. II, No. 3. 
The observed right ascensions at the epoch and the equinox of the observation have been reduced to the mean epoch and the equinox 1950.0, by basing Newcomb's precession constants in accordance with the FK3 system.

The total mean value of $\Delta \alpha$ of all clock stars is -0.0024 , and their mean value of each hour of right ascension (overlapped for each three hours) is shown as the black points in Fig. 1. In Fig. 1, the white points show the $\Delta \alpha$ in the ecliptic zone obtained from the observations by the same writer ${ }^{2)}$. The ecliptic zone has been observed very frequently in the past and also in the present century, while the zenith zone of our observatory has not been observed so frequently. Fig. 1 shows that the deviations of the positions of clock stars in the zenith zone are larger than those of ecliptic. The writer hopes that the improvement of the star positions in the present catalogue may be continued in future in view of its import ance as the standard system of the clock stars of our observatory.

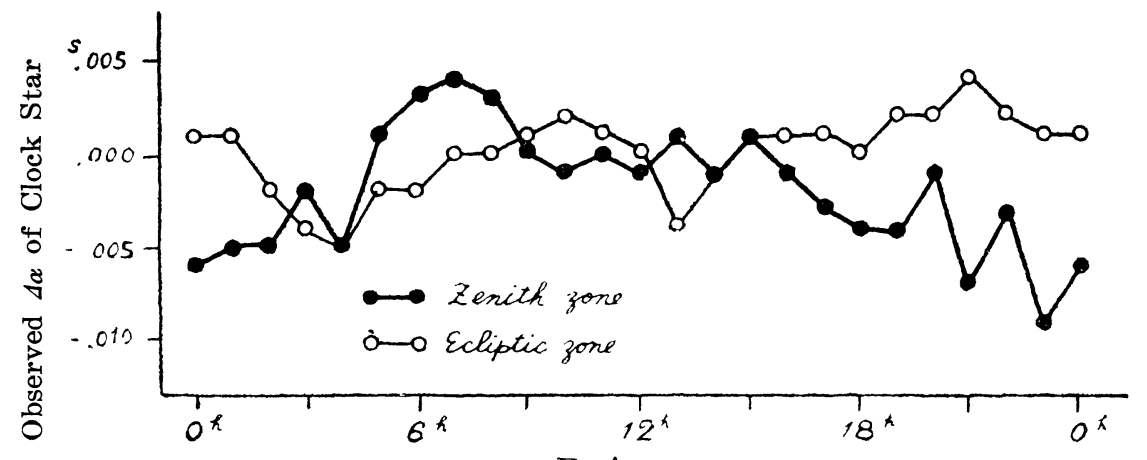

R. A.

Fig. 1.

3. The determination of the proper motions of the zenith stars.

The program stars of zenith zone have been selected from GC and AG catalogues. To obtain their proper motions in accordance with tne FK3 system, we must determine the catalogue difference between FK3, GC and AG. The positions of 100 clock stars are designated in FK3, GC and AG catalogues. Comparing those data, we obtain the catalogue difference between those catalogues in the zenith zone of our observatory as in Table I.

Applying the above corrections to the position of the zenith stars in GC and AG catalogues, we obtain the former normal positions which have been reduced to the FK3 system. Comparing our

2) Tokyo-Mitaka Catalogue of Zodiacal Stars. Annals of the Tokyo Astronomical Observatory, Second Series, Vol. II, No. 1. 
observed right ascensions with those former normal positions, we obtain the proper motions of the observed zenith stars.

Table I

Catalogue difference in the zenith zone at Tokyo-Mitaka.

$\Delta x=x+y \sin \alpha+z \cos \alpha+u \sin 2 x+u \cos 2 a$.

\begin{tabular}{|c|c|c|c|c|c|c|}
\hline $\begin{array}{c}\text { Cata- } \\
\text { logues }\end{array}$ & Epoch & $x$ & $y$ & $z$ & $w$ & $u$ \\
\hline FK3-GC & GC & $\begin{array}{c}\mathrm{s} \\
-.0103 \pm .0003\end{array}$ & 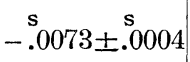 & $\begin{array}{c}s \\
+.0079 \pm .0004\end{array}$ & $\begin{array}{c}\mathrm{s} \\
-.0024 \pm .0004\end{array}$ & $\begin{array}{l}\mathrm{s} \\
+.0031 \pm .0004\end{array}$ \\
\hline FK3-AG & $\mathrm{AG}$ & $\mathrm{s} .0636 \pm .0023$ & $-\mathrm{s} .0074 \pm .0034$ & $\begin{array}{l}\mathrm{s} \\
+.0045 \pm .0034\end{array}$ & $\begin{array}{l}\mathrm{s} \\
+.0025 \pm .0033\end{array}$ & $-.0060 \pm .0034$ \\
\hline
\end{tabular}

4. The catalogue difference between (Tokyo-Mitaka)-(GC).

We have a group of 500 zenith stars in the present catalogue of which the positions are also designated in GC catalogue at the epoch and the equinox 1950.0. The catalogue difference of the positions of those stars between these two catalogues is obtained and shown with the black points and the full line in Fig. 2. On the other hand the catalogue difference between (FK3)-(GC) in Table I is shown with the broken line in Fig. 2.

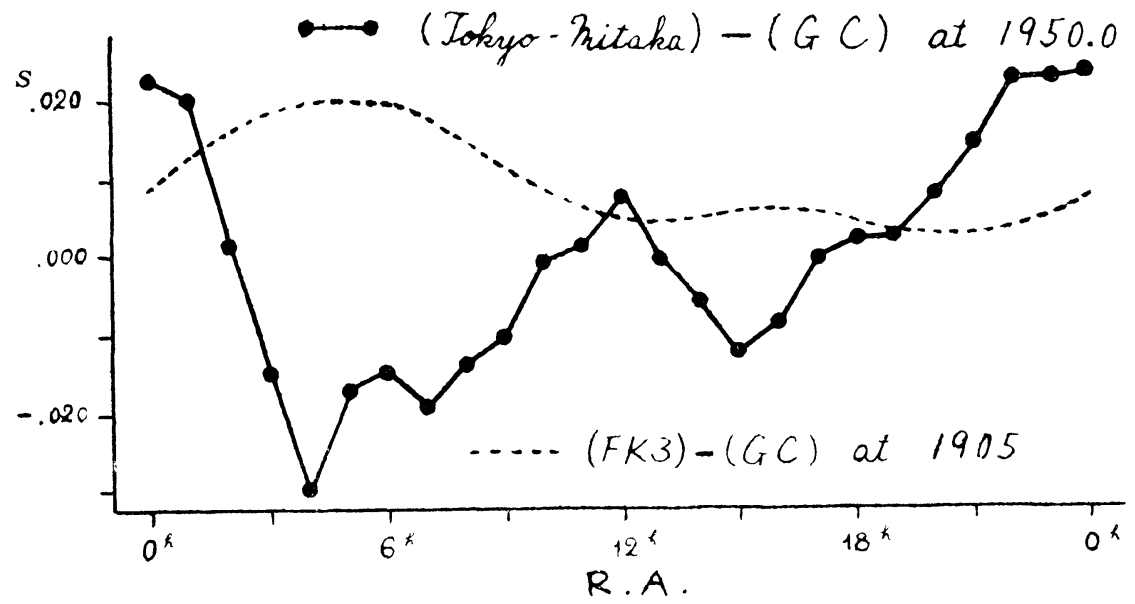

Fig. 2. The catalogue difference in R. A.

The broken line in Fig. 2 has been derived from the clock stars of which the magnitudes are brighter than the program stars, and its epoch is $1900 \sim 1910$. The full line in Fig. 2 has been derived from rather faint stars compared with the clock stars. Therefore the difference of the two curves in Fig. 2 may be speculated as the results of the motions of the two different groups of stars for half a century. 
But the writer wants to introduce the catalogue difference between Tokyo-Mitaka Zodiacal Catalogue and GC catalogue. In Fig. 3 we overlap it with the phase-difference of 3 hours on the full line in Fig. 2. The similarity of the two curves shows the presence of the same tendency for $\Delta a_{x}$, but the appearance of larger amplitude for $\Delta \alpha_{\alpha}$ in the ecliptic zone may be due to the fact that more faint stars have been observed in ecliptic than in zenith zone.

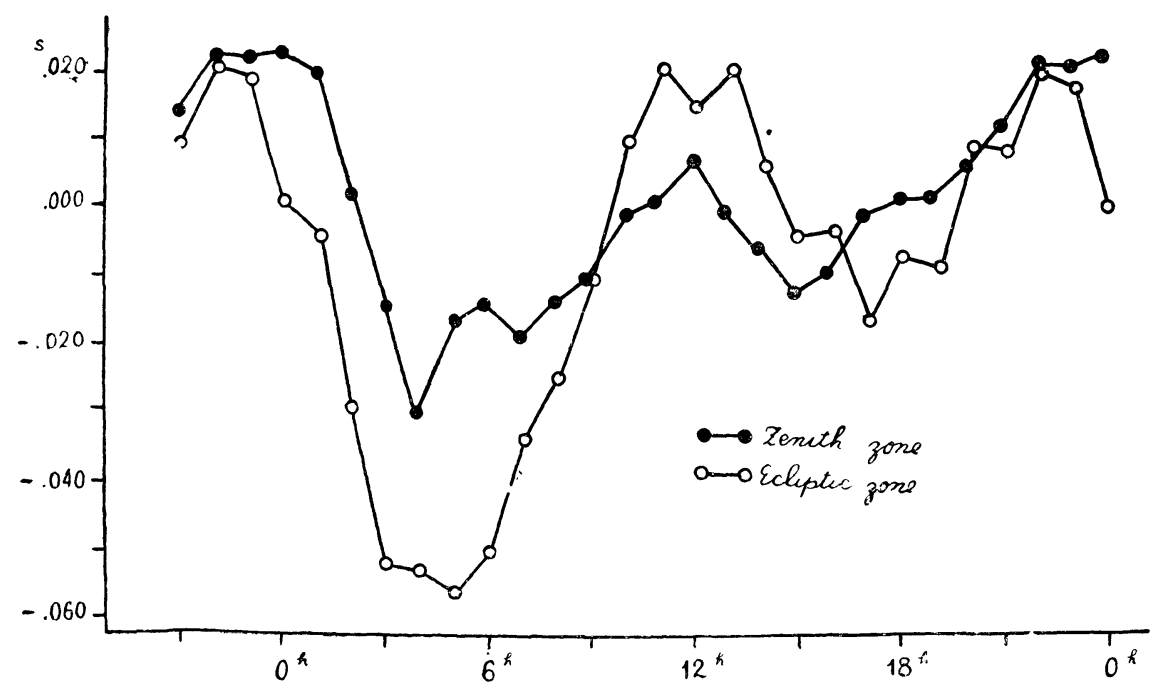

R. A.

Fig. 3. Catalogue difference between.

(Tokyo-Mitaka Zenith) and GC at $\mathbf{1 9 5 0 . 0}$

(Tokyo-Mitaka Ecliptic) and GC at 1950.0

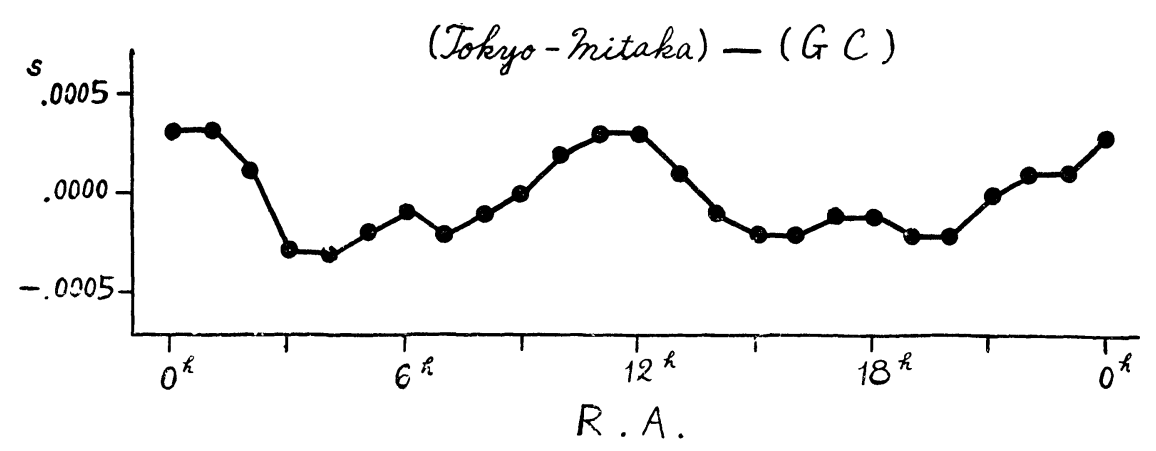

Fig. 4. The catalogue difference in $\Delta u$.

The catalogue difference of the proper motions between the present catalogue and GC is shown in Fig. 4. It has also something of similarity to that for the ecliptic zone obtained in the writer's former observation. In the latter case, the writer has discussed 
it as the result of a small variation in the second term of the obliquity of ecliptic ${ }^{3)}$.

In the zenith zone, the catalogue difference of the proper motions arranged for right ascension in Fig. 4 may be expressed in the form of $x+y \sin (2 \alpha+\theta)$, but its amplitude is one-tenth of the corresponding one in the ecliptic zone. Its complete interpretation may be more difficult in the zenith zone, and the writer wishes to reserve it for future research.

3) Proc. Japan Acad. Vol, XXI, No. 3, p. 192. 